\title{
Massless BPS-Saturated States on the Two-Torus Moduli Sub-Space of Heterotic String
}

\author{
Kwan-Leung Chan ${ }^{1}$ and Mirjam Cvetič ${ }^{2}$ * \\ 1 Department of Physics and Astronomy \\ University of Pennsylvania, Philadelphia PA 19104-6396, \\ 2 Institute for Advanced Study \\ Olden lane, Princeton, NJ 08540
}

(December 1995)

\begin{abstract}
Within a four-dimensional, toroidally compactified heterotic string, we identify (quantized) charge vectors of electrically charged BPS-saturated states (along with the tower of $S L(2, Z)$ related dyonic states), which preserve $\frac{1}{2}$ of $N=4$ supersymmetry and become massless along the hyper-surfaces of enhanced gauge symmetry of the two-torus moduli sub-space. In addition, we identify charge vectors of the dyonic BPS-saturated states (along with the tower of $S L(2, Z)$ related states), which preserve $\frac{1}{4}$ of $N=4$ supersymmetry, and become massless at two points with the maximal gauge symmetry enhancement.
\end{abstract}

* On sabbatic leave from the University of Pennsylvania. 
BPS-saturated states of four-dimensional, toroidally compactified heterotic string theory provide a fruitful ground to address non-perturbative aspects of string theory. In particular, regular solutions, i.e., those with regular horizons, may shed light on quantum aspects of black hole physics, e.g., on statistical interpretation of black hole entropy [1]:2] while those that can become massless [3 5 ] at certain points of moduli space may shed light on the nature of enhanced symmetries [6.4] at special points of moduli space. Since the effective theory possessed $N=4$ supersymmetry, the ADM mass for these BPS-saturated states is protected from quantum corrections. In principle, one should be able to trust the BPS mass formula even in the case where quantized charges are of $\mathcal{O}(1)$.

In this letter we further address massless BPS-saturated states. In particular, we identify the (quantized) charge vectors and the points (lines, hyper-surfaces) in the moduli space for which the BPS-saturated states become massless. For the sake of simplicity we confine this study to the two-torus moduli sub-space. The work generalizes that of Ref. [4], where the case of the two-circle moduli sub-space was addressed.

Recently, the explicit form of the generating solution [2:7] for all the static, spherically symmetric BPS-saturated states in this class was obtained. The generating solution is specified by five (electric and magnetic) charges of the two $U(1)_{a, b}$ Kaluza-Klein and two $U(1)_{c, d}$ two-form fields associated with the two, say the first two, (toroidally) compactified dimensions. The most general BPS-saturated state in this class is parameterized by unconstrained 28 electric and 28 magnetic charges and is obtained by applying a subset of $T$-duality and $S$-duality transformations, which do not affect the four-dimensional space-time, on the generating solution.

The ADM mass for these states (BPS mass formula), which in general preserve only $\frac{1}{4}$ of supersymmetry, is specified [8,9], in terms of 28 electric and 28 magnetic charges. For the purpose of studying the moduli (and the dilaton-axion) dependence of the BPS mass formula [8,9] we rewrite it in terms of conserved magnetic $(\vec{\beta})$ and electric $(\vec{\alpha})$ charge vectors [2]: []

$$
M_{B P S}^{2}=\frac{1}{2} e^{-2 \phi_{\infty}} \vec{\beta}^{T} \mu_{R} \vec{\beta}+\frac{1}{2} e^{2 \phi_{\infty}} \overrightarrow{\tilde{\alpha}}^{T} \mu_{R} \overrightarrow{\tilde{\alpha}}+\left[\left(\vec{\beta}^{T} \mu_{R} \vec{\beta}\right)\left(\vec{\alpha}^{T} \mu_{R} \vec{\alpha}\right)-\left(\vec{\beta}^{T} \mu_{R} \vec{\alpha}\right)^{2}\right]^{\frac{1}{2}},
$$

where

$$
\overrightarrow{\tilde{\alpha}} \equiv \vec{\alpha}+\Psi_{\infty} \vec{\beta}, \quad \mu_{R, L} \equiv M_{\infty} \pm L .
$$

The charge vectors $\vec{\alpha}$ and $\vec{\beta}$ are related to the physical magnetic $\vec{P}$ and electric $\vec{Q}$ charges in the following way:

$$
\sqrt{2} P_{i}=L_{i j} \beta_{j}, \quad \sqrt{2} Q_{i}=e^{2 \phi_{\infty}} M_{i j \infty}\left(\alpha_{j}+\Psi_{\infty} \beta_{j}\right), \quad(i=1, \cdots, 28)
$$

\footnotetext{
${ }^{1}$ In Ref. [7] also all the non-extreme solutions were obtained. In Ref. [2] it was shown that BPSsaturated generating solution is an exact target-space background solution of a conformal $\sigma$-model.

${ }^{2}$ We use the notation and conventions, as specified in Refs. [7], following e.g., Ref. [10,11].
} 
where the subscript $\infty$ refers to the asymptotic $(r \rightarrow \infty)$ value of the corresponding fields. Here, the moduli matrix $M$ and the dilaton-axion field $S \equiv \Psi+i \mathrm{e}^{-2 \phi}$, transform covariantly (along with the corresponding charge vectors) under the $T$ - duality $(O(6,22, Z))$ and $S$ duality $\left(S L(2, Z)_{S}\right)$, respectively, while the BPS mass formula (1) remains invariant under these transformations.

Note that when the magnetic and electric charges are parallel in the $O(6,22)$ sense, i.e., $\vec{\beta} \propto \vec{\alpha}$ (in the quantized theory the lattice charge vectors should be relative co-primes [11]), the BPS mass formula (1) is that of the BPS-saturated states which preserve $\frac{1}{2}$ of $N=4$ supersymmetry (see, e.g., [11]). In the case when the magnetic and electric charges are not parallel, the mass is larger (the last term in (11) is non-zero) and the configurations preserve only $\frac{1}{4}$ of $N=4$ supersymmetry [8]. Note that states preserving $\frac{1}{2}$ of $N=4$ supersymmetry belong to the vector super-multiplets, while those preserving $\frac{1}{4}$ of $N=4$ supersymmetry belong to the highest spin $\frac{3}{2}$-supermultiplets [12,13]. Thus, when the former [latter] states become massless they may contribute to the enhancement of gauge symmetry [6] [supersymmetry [4]].

In the quantum theory the charge vectors $\vec{\alpha}, \vec{\beta}$ are quantized. Following [11, one may attempt to constrain the allowed lattice charge vectors by using the constraints for the elementary BPS-saturated string states of toroidally compactified heterotic string, along with the Dirac-Schwinger-Zwanziger-Witten (DSZW) [14] quantization condition.[1 Purely electric BPS-saturated states $(\vec{\beta}=0)$ preserve $\frac{1}{2}$ of $N=4$ supersymmetry and have the same quantum numbers [15,16] as the elementary BPS-saturated string states with no excitations in the right-moving sector $\left(N_{R}=\frac{1}{2}\right)$. For the electric states the quantized charge vector $\vec{\alpha}$ is then constrained to lie on an even self-dual lattice $\Lambda_{6,22}$ with the following norm (in the $O(6,22)$ sense) [11:

$$
\vec{\alpha}^{T} L \vec{\alpha}=2 N_{L}-2=-2,0,2, \ldots
$$

where the integer $N_{L}$ parameterizes the level of the left-moving sector.

The DSZW charge quantization condition then implies an analogous constraint for $\vec{\beta}^{T} L \vec{\beta}$; magnetic charge vectors $\vec{\beta}$ are then constrained [11] to lie on an even self-dual lattice $\Lambda_{6,22}$ with the norm

$$
\vec{\beta}^{T} L \vec{\beta}=2 N_{L}-2=-2,0,2, \ldots .
$$

Since we confine the analysis to the two-torus moduli sub-space, the $T$-duality group reduces to $O(2,2)$. Then only the $O(2,2)$ part of the symmetric moduli metric $M$ is non-trivial and of the form:

$$
M=\left(\begin{array}{cc}
G^{-1} & -G^{-1} B \\
-B^{T} G^{-1} & G+B^{T} G^{-1} B
\end{array}\right), \quad L=\left(\begin{array}{cc}
0 & I_{2} \\
I_{2} & 0
\end{array}\right)
$$

where $G \equiv\left[G_{m n}\right]((m, n)=1,2), B \equiv B_{12}$ are the four moduli of the two-torus and $L$ is an $O(2,2)$ invariant matrix.

\footnotetext{
${ }^{3}$ In Ref. [2] the charge quantization for the generating solution is implied by considering the conformal field theory describing the throat region of the corresponding string solution.
} 
The four moduli fields can be expressed in terms of two complex fields $T$ and $U$, (see, e.g., 17] and references therein):

$$
T \equiv \sqrt{\mathcal{G}}+i B, \quad U \equiv \frac{\sqrt{\mathcal{G}}-i G_{12}}{G_{11}}
$$

where $\mathcal{G} \equiv \operatorname{det}\left(G_{m n}\right)$. The $T$ and $U$ fields transform covariantly under $P S L(2, Z)_{T}$ and $P S L(2, Z)_{U}$, respectively, i.e., the subgroups of the duality group $O(2,2, Z)=P S L(2, Z)_{T} \times$ $P S L(2, Z)_{U} \times Z_{2 T \leftrightarrow U}$ [17].

In order to address massless BPS states which preserve $\frac{1}{2}$ of supersymmetry, we first concentrate on purely electrically charged configurations $(\vec{\beta}=0)$ with the electric lattice charge vector $\vec{\alpha} \equiv\left(\alpha_{a}, \alpha_{b} ; \alpha_{c}, \alpha_{d}\right)$ whose norm is:

$$
\vec{\alpha}^{T} L \vec{\alpha}=-2
$$

Namely, only the states with the electric charge norm (8) can become massless [6] along the lines (hyper-surfaces) of moduli space for which:

$$
\vec{\alpha}^{T} \mu_{R} \vec{\alpha}=0
$$

It turns out that (9) is satisfied along the following hyper-surfaces, along with the following accompanying electric charge vectors $\vec{\alpha}$ : :

$$
\begin{gathered}
\mathcal{L}_{1}: U=T \Leftrightarrow\left(G_{11}, G_{22}, G_{12}, B\right)=\left(1, G_{22},-B, B\right) ; \quad \vec{\alpha}=\vec{\lambda}_{1 \pm} \equiv \pm(1,0,-1,0), \\
\mathcal{L}_{2}: U=\frac{1}{T} \Leftrightarrow\left(G_{11}, G_{22}, G_{12}, B\right)=\left(G_{11}, 1, B, B\right) ; \quad \vec{\alpha}=\vec{\lambda}_{2 \pm} \equiv \pm(0,1,0,-1), \\
\mathcal{L}_{3}: U=T-i \Leftrightarrow\left(G_{11}, G_{22}, G_{12}, B\right)=\left(1, G_{22}, 1-B, B\right) ; \quad \vec{\alpha}=\vec{\lambda}_{3 \pm} \equiv \pm(1,1,-1,0), \\
\mathcal{L}_{4}: U=\frac{T}{i T+1} \Leftrightarrow\left(G_{11}, G_{22}, G_{12}, B\right)=\left(G_{11},-1+2 B+G_{11},-1+B+G_{11}, B\right) ; \\
\vec{\alpha}=\vec{\lambda}_{4 \pm} \equiv \pm(1,0,-1,1),
\end{gathered}
$$

Those are the same four hype-surfaces of the two-torus moduli sub-space (in the fundamental domain), for which the gauge symmetry of toroidally compactified heterotic string is enhanced due to the Halpern-Frenkel-Kač mechanism, i.e., those are the hyper-surfaces where the perturbative string states (with $N_{R}=\frac{1}{2}$ ), which have the same quantum numbers as electrically charged BPS-saturated states, become massless. Thus, on the heterotic side these electrically charged BPS-states are identified with the elementary string excitations.

\footnotetext{
${ }^{4}$ In the following we suppress the subscript $\infty$ for the asymptotic values of the moduli fields.
} 
Along each of the hyper-surfaces $\mathcal{L}_{1,2,3,4}$ these electrically charged massless BPS-saturated states, which are scalar components of the vector super-multiplets, contribute to the enhancement of the gauge symmetry from $\left[U(1)_{a} \times U(1)_{b} \times U(1)_{c} \times U(1)_{d}\right]$ (at generic points of moduli space) to $\left[U(1)_{b} \times U(1)_{d} \times U(1)_{a+c} \times S U(2)_{a-c}\right],\left[U(1)_{a} \times U(1)_{c} \times U(1)_{b+d} \times S U(2)_{b-d}\right]$, $\left[U(1)_{d} \times U(1)_{a+c} \times U(1)_{a-2 b-c} \times S U(2)_{a+b-c}\right]$ and $\left[U(1)_{b} \times U(1)_{a+c} \times U(1)_{a-c-2 d} \times S U(2)_{a-c+d}\right]$, respectively.

At the point $U=T=1$, i.e., $\left(G_{11}, G_{22}, G_{12}, B\right)=(1,1,0,0)$ (the self-dual point of the two-circle), $\mathcal{L}_{1}$ and $\mathcal{L}_{2}$ meet and the enhanced gauge symmetry is $\left[U(1)_{a+c} \times U(1)_{b+d} \times\right.$ $\left.S U(2)_{a-c} \times S U(2)_{b-d}\right]$. At the point $T=U^{*}=e^{i \frac{\pi}{6}}$, i.e., $\left(G_{11}, G_{22}, G_{12}, B\right)=\left(1,1, \frac{1}{2}, \frac{1}{2}\right)$, $\mathcal{L}_{2}, \quad \mathcal{L}_{3}$ and $\mathcal{L}_{4}$ meet and the enhanced gauge symmetry is $\left[U(1)_{a+c} \times U(1)_{a-2 b-c-2 d} \times\right.$ $\left.S U(3)_{b-d, 2 a+b-2 c+d}\right]$. Here the subscript(s) for the non-Abelian gauge factors $(S U(2), S U(3))$ denote the linear combinations of the Abelian generators that determine the diagonal generator(s) of the non-Abelian factors.

Due to the $S L(2, Z)_{S}$ symmetry, along with each of the charge vectors $\vec{\alpha}$ (as specified in (10)-(13) ), there is a tower of dyonic configurations (including the $Z_{2}$ related purely magnetic states) with $p \vec{\beta}=q \vec{\alpha}$, where $p$ and $q$ are the relative co-primes [11]. These dyonic configurations become massless at the same points of moduli space as purely electric configurations.

We now address massless dyonic states whose electric and magnetic charge vectors are not parallel. These states only preserve $\frac{1}{4}$ of $N=4$ supersymmetry. The necessary condition for them to become massless is that both the electric and the magnetic charge vector norms satisfy:

$$
\vec{\alpha}^{T} L \vec{\alpha}=-2, \quad \vec{\beta}^{T} L \vec{\beta}=-2 .
$$

These BPS-saturated states become massless at the points of moduli space for which now the following three constraints are satisfied:

$$
\vec{\alpha}^{T} \mu_{R} \vec{\alpha}=0, \quad \vec{\beta}^{T} \mu_{R} \vec{\beta}=0, \quad \vec{\beta}^{T} \mu_{R} \vec{\alpha}=0,
$$

By explicit calculation we found that the three constraints (15) are satisfied only at the following two points:

$$
\begin{gathered}
T=U=1, \quad(\vec{\alpha}, \vec{\beta})=\left(\vec{\lambda}_{1 \pm}, \vec{\lambda}_{2 \pm}\right), \\
T=U^{*}=e^{i \frac{\pi}{6}}, \quad(\vec{\alpha}, \vec{\beta})=\left(\vec{\lambda}_{i \pm}, \vec{\lambda}_{j \pm}\right), \quad[(i, j)=2,3,4, \quad i<j] .
\end{gathered}
$$

The charge assignments for the four massless dyonic BPS-saturated states (16) at the selfdual point of the two-circle were found in Ref. [4]. At the point $T=U^{*}=e^{i \frac{\pi}{6}}$ there are twelve massless dyonic BPS-saturated states (17). In addition, there is an infinite $S L(2, Z)_{S}$ related tower of massless states (including the $Z_{2}$ related states with electric and magnetic charge vectors in (16) and (17) interchanged). Since these states belong to the highest spin $\frac{3}{2}$-supermultiplet, they may contribute to the enhancement of supersymmetry there. Note that dyonic states (16) and (17) are not in the perturbative spectrum of toroidally compactified heterotic string. 
A few comments are in order. The discussed BPS-saturated states become massless at special points and hyper-surfaces of moduli space, regardless of the strength of the dilatonaxion coupling. Note also that all the discussed states are singular four-dimensional solutions. Namely, for the solutions to be regular, i.e., with the (Einstein frame) event horizon, the norms of the lattice charge vectors have to satisfy the following constraints [2]:

$$
\vec{\alpha}^{T} L \vec{\alpha}>0, \quad \vec{\beta}^{T} L \vec{\beta}>0, \quad\left(\vec{\beta}^{T} L \vec{\beta}\right)\left(\vec{\alpha}^{T} L \vec{\alpha}\right)-\left(\vec{\beta}^{T} L \vec{\alpha}\right)^{2}>0 .
$$

Since the norms (8), (14) of the massless BPS states are negative, all the above solutions are singular from the four-dimensional point of view.

The above solutions were obtained as semi-classical solutions of toroidally compactified heterotic string; they are parameterized in terms of classical bosonic fields of heterotic string and (quantized) lattice charge vectors, consistent with the heterotic string constraints and the DSZW quantization condition. It is important to address the stability of these configurations, as well as to identify these semi-classical solutions in terms of the $D$-brane [18] solutions of Type IIA string.

After the results presented in the paper had been obtained, we became aware of the paper [19] where related issues were addressed.

\section{ACKNOWLEDGMENTS}

The work was supported in part by U.S. Department of Energy Grant No. DOE-EY-7602-3071, the Institute for Advanced Study funds and J. Seward Johnson foundation (M.C.), and the National Science Foundation Career Advancement Award PHY95-12732 (M.C.). M.C. would like to thank D. Youm for discussions.

\footnotetext{
${ }^{5}$ Note, that the BPS mass formula (1) is semi-positive definite for any asymptotic value of the axion field $\Psi_{\infty}$. This result is due to the fact that the lattice vectors satisfy $\vec{\alpha}^{T} \mu_{R} \vec{\alpha} \geq 0, \quad \vec{\beta}^{T} \mu_{R} \vec{\beta} \geq 0$, and $\left(\vec{\alpha}^{T} \mu_{R} \vec{\alpha}\right)\left(\vec{\beta}^{T} \mu_{R} \vec{\beta}\right)-\left(\vec{\beta}^{T} \mu_{R} \vec{\alpha}\right)^{2} \geq 0$ everywhere in the moduli space.
} 


\section{REFERENCES}

[1] A. Sen, Mod. Phys. Lett. A10 (1995), 2081, hep-th/9504147; A. Peet, Nucl. Phys. B456 (1995) 732, hep-th/9506200; F. Larsen and F. Wilczek, PUPT-1576, hep-th/9511064.

[2] M. Cvetič and A.A. Tseytlin, IASSNS-HEP-95-102, hep-th/9512031.

[3] K. Behrndt, Nucl. Phys.B455 (1995) 188, hep-th/9506106 R. Kallosh, Phys. Rev. D52 (1995) 6020, hep-th/9506113; R. Kallosh and A. Linde, Phys. Rev. D52 (1995) 7137, hep-th/9507022.

[4] M. Cvetič and D. Youm, Phys. Lett. B359 (1995) 87, hep-th/9507160.

[5] R. Kallosh and A. Linde, SU-ITP-95-26, hep-th/9511115.

[6] C.M. Hull and P.K. Townsend, Nucl. Phys. B451 (1995) 525, hep-th/9505073.

[7] M. Cvetič and D. Youm, IASSNS-HEP-95/107, hep-th/9512127.

[8] M. Cvetič and D. Youm, Phys. Rev. D53 (1996) R584, hep-th/9507090.

[9] M.J. Duff, J.T. Liu and J. Rahmfeld, CTP-TAMU-27-95, hep-th/9508094.

[10] J. Maharana and J.H. Schwarz, Nucl. Phys. B390 (1993) 3, hep-th/9207016.

[11] A. Sen, Int. J. Mod. Phys. A9 (1994) 3707, hep-th/9402002.

[12] J. Strathdee, Int. J. Mod. Phys. A2 (1987) 273.

[13] R. Kallosh, Phys. Rev. D52 (1995) 1234, hep-th/9503029.

[14] E. Witten, Phys. Lett. B86 (1979) 283.

[15] M.J. Duff and J. Rahmfeld, Phys. Lett. B345 (1995) 441, hep-th/9406105.

[16] C.G. Callan, J.M. Maldacena and A.W. Peet, PUPT-1565, hep-th/9510134; A. Dabholkar, J.P. Gauntlett, J.A. Harvey and D. Waldram, CALT-68-2028, hep-th/9511053.

[17] A. Giveon, M. Porrati and E. Rabinovici, Phys. Rep. 244 (1994) 77, hep-th/9401139.

[18] J. Polchinski, Phys. Rev. Lett. 75 (1995) 4724-4727, hep-th/9510017.

[19] G. Lopes Cardoso, G. Curio, D. Lüst, T. Mohaupt and S-J. Rey, HUB-EP-95-33, hepth/9512129. 